\title{
Field large-scale relative density tests of gravel soil of Yulongkashi high earth-rock fill dam
}

\author{
Yang Zhengquan ${ }^{1,2, a}$, Xue Yijie ${ }^{1,3, b}$, Wang Long ${ }^{1,2, c}$, Liu Xiaosheng ${ }^{1,2, d}$ \\ Liu Qiwang ${ }^{1,2, e}$ and Zhao Jianming ${ }^{1,2, f}$
}

\author{
${ }^{1}$ State Key Laboratory of Simulation and Regulation of Water Cycle in River Basin, Beijing 100038 \\ ${ }^{2}$ China Institute of Water Resources and Hydropower Research, Beijing 100048 \\ ${ }^{3}$ College of Water Resources and Civil Engineering, China Agricultural University, Beijing 100083 \\ ayangzhq@iwhr.com, b1277117693@qq.com, c345335499@qq.com, dliuxsh@iwhr.com,
}

eliuqw@iwhr.com, 'zhaojm@iwhr.com Keywords: Gravel soil. Relative density. Field test. Optimum coarse grain content. Three factors
chart

Abstract. The compaction rate of gravel soil is usually characterized by the "relative density". In order to calculate the relative density of gravel soil, the relative density property index of soil should be determined firstly, such as the maximum dry density and the minimum dry density. Because of the size and energy limitation of the laboratory test equipment, The maximum dry density of the gravel soils determined in laboratory tests is often much lower than the actual. For the Yulongkashi high earth-rock fill dam in China, the field large-scale relative density tests of original gravel soil was carried out used actual rolling equipment and large relative density bucket, the compaction characteristics of soil was studied, and the relative density characteristics index of soils with different gradation (coarse grain content) were determined. The test results showed that, compared with the general laboratory test results, the maximum dry density of gravel soil determined by field test was much larger; the minimum dry density as well as maximum dry density of gravel soil had the tendency of increasing and then decreasing with the increase of coarse grain content, and there was an optimum coarse grain content for the density of soil.

\section{Introduction}

The relative density is generally used to measure the compaction rate of gravel soil for dam material $^{[1]}$, which is used as the criterion of dam design and filling density to quantitatively evaluate the compaction quality of dam construction. The premise of determining the relative density of gravel soil in a given state is that the maximum as well as minimum dry density are known, but the maximum as well as minimum dry density with different gradation or coarse grain content $\left(\mathrm{P}_{5}\right)$ are different. Coarse grain content $\left(\mathrm{P}_{5}\right)$ refers to the proportion of soil particle size larger than $5 \mathrm{~mm}$ in total soil weight. At present, to determine the maximum as well as minimum dry density of gravel soil with different gradation (coarse grain content), laboratory relative density test is widely used. Because the maximum particle size of the prototype gravel soil often exceeds the size limit of the laboratory test equipment, the laboratory test is usually to scale the prototype gravel soil according to certain rules and obtain the actual test soil material ${ }^{[2]}$. Due to the influence of the size of laboratory test equipment and the vibration compaction equipment energy, the maximum dry density of the soil determined by laboratory test is often much lower than the actual value. If used directly without modification, it is often observed that the relative density of gravel soil compaction tested in the field is greater than $1^{[3]}$. As far as the present research is concerned, there is no widely accepted method for determining the maximum dry density of the actual dam material in the field.

It is an effective way to solve the problem that the above laboratory test results can not reflect the actual engineering problems by using the actual construction rolling machine and large relative density bucket at the scene to test the maximum and minimum dry density of original gravel soil ${ }^{[3]}$. Large relative density buckets and corollary equipment are used to carry out the test, which ensures that the original gravel soil can be tested directly, thus eliminating or reducing the error caused by the size effect; it can reflect the actual condition of the field compaction construction and overcome 
the shortcoming of insufficient compaction energy in the laboratory test by using the actual construction rolling machine as the vibration compaction equipment of the relative density test. The existing research results show that the maximum and minimum dry density index of gravel soil can be determined by field large-scale relative density test, which can contain the measured roller dry density of dam material obtained actual construction inspection, and the results of field large-scale relative density test are reliable, it can be used as the evaluation basis for the quality of gravel soil compaction construction.

The maximum dam height of the Yulongkashi earth-rock fill dam in China is more than $200 \mathrm{~m}$, the gravel soil was used as the main material for the dam. In view of the fact that the compactness of gravel soil had an important influence on its engineering characteristics ${ }^{[4]}$, to study the relative density (compaction) characteristics of gravel soil, to determine the control standard of dam filling density and to determine the construction rolling parameters, which were of great significance to ensure the filling quality of the dam and the later operation safety. In this paper, at the scene of dam material the relative density test of gravel soil of Yulongkashi high earth-rock fill dam was carried out, and the maximum as well as minimum dry density index of gravel soil with different gradation (coarse grain content) was determined, which was the basis for evaluating the compaction quality of gravel soil.

\section{Text methods and procedures}

Large-scale relative density test equipment mainly included: actual construction rolling machine was a 26 ton smooth drum vibrating roller used in subsequent dam rolling construction; density buckets were steel barrels without lid, $120 \mathrm{~cm}$ in diameter, $80 \mathrm{~cm}$ in height, $1.2 \mathrm{~cm}$ in thickness, the density buckets could be used for the original gradation test of dam material, and the effect of size effect was basically eliminated; equipment for measuring dry density of coarse grained soil; corollary equipment of construction and soil screening equipment, etc.

Selected a smooth and solid site for the relative density test site. Dug a trench 2.5 meters wide, 15.0 meters long and 1.2 meters deep, the bottom of the trench was leveling and then compacted with smooth drum vibrating roller; arranged 5 exactly the same density buckets side by side in the trench; filled with soil of similar properties to test soil around density buckets and then vibration rolling, ensured that the density bucket was fastened to the site. The density buckets and test sites after installation are shown in the figure1.

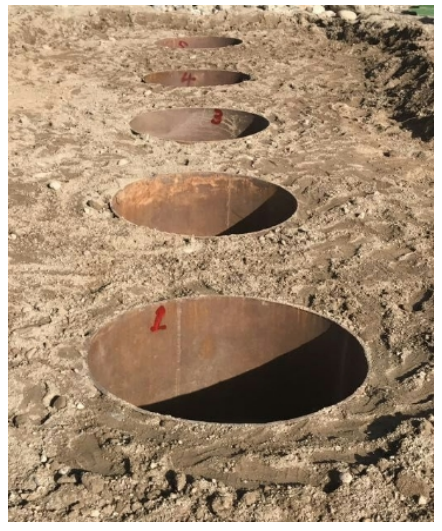

Fig.1 Placement of density buckets

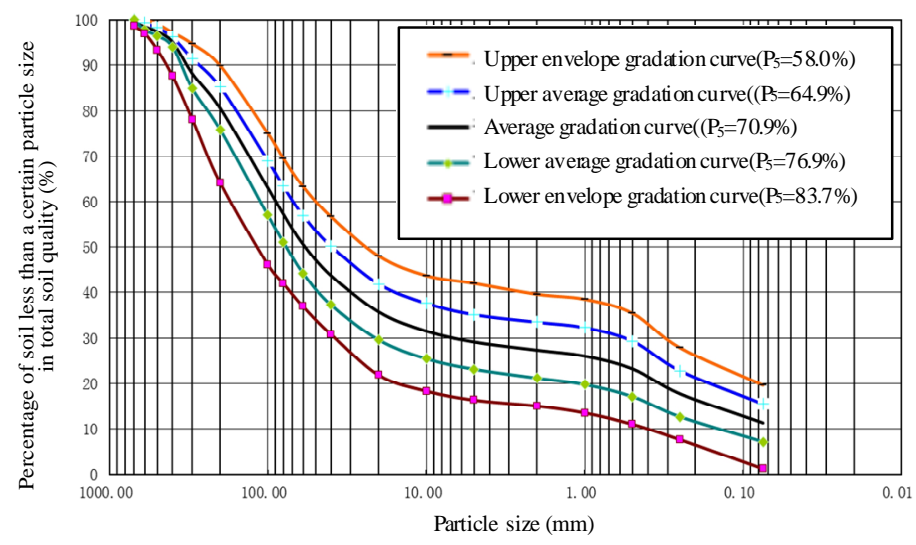

Fig.2 Gradation curve of test soil

The characteristic gradation curves of gravel soil used in the design include: average gradation curve; upper envelope gradation curve; lower envelope gradation curve; upper average gradation curve and lower average gradation curve, five kinds of characteristic gradation were also used to characterize five different kinds of coarse grain content. Accorded to the above 5 kinds of characteristic gradation, the test soil was made by artificial preparation after screened with prototype gravel soil in the field. After the test of five kinds of characteristic gradation (coarse grain content) soil was finished, accorded to the optimum coarse grain content determined by the test, the optimum coarse grain content soil material was confected again to carry on the check test. The 
results of subsequent tests showed that the optimum coarse grain content of gravel soil in Yulongkashi was basically the same as the design lower average gradation curve, so the design lower average gradation curve was used to calibrate the test soil materials. The test soil gradation curves is shown in figure2.

First of all, the minimum dry density test was carried out by using the density bucket; after the minimum dry density test was completed, the soil was replenished, the maximum dry density of soil was determined by vibration rolling with actual construction rolling machine.

Minimum dry density test used artificial loose filling method: weighed the prepared soil and filled it evenly and loosely in density buckets, when filling, the soil was light placed in the buckets to prevent shock and vibration, so as to simulate the natural accumulation of soil as much as possible; when the soil was loosely filled to the top of the buckets, the top surface of the bucket should was leveled with a rigid leveling tool, and the disturbed soil sample should was avoided as far as possible; after filling, the total weight of the fill in the buckets was recorded, and the minimum dry density was calculated according to the filling weight and the volume of the bucket. The loose filling method process is shown in figure3.

After the minimum dry density test was completed, the maximum dry density test was carried out: spread the residual soil of the minimum dry density test on the surface of the density bucket to a certain extent, the surface of the tiled soil was about $20 \mathrm{~cm}$ above the top of the bucket, ensured site leveling after spreading of soil; the smooth drum vibrating roller started off the field, and the speed was controlled within $2 \mathrm{~km} / \mathrm{h}$, after 26 times of strong vibration rolling in the upper region of the density bucket, then 15 minutes of strong vibration rolling at low speed back and forth within each density bucket, in the process of compaction, the soil material should be replenished in time, so that the smooth drum vibrating roller would not be in direct contact with density buckets; after the strong vibration rolling was completed, the excess soil above the top of the buckets was removed and leveled; dug out all the soil in the bucket and weigh it, calculated the maximum dry density according to the total soil weight and the volume of the bucket. The strong vibration rolling and density bucket leveling is shown in figure4.

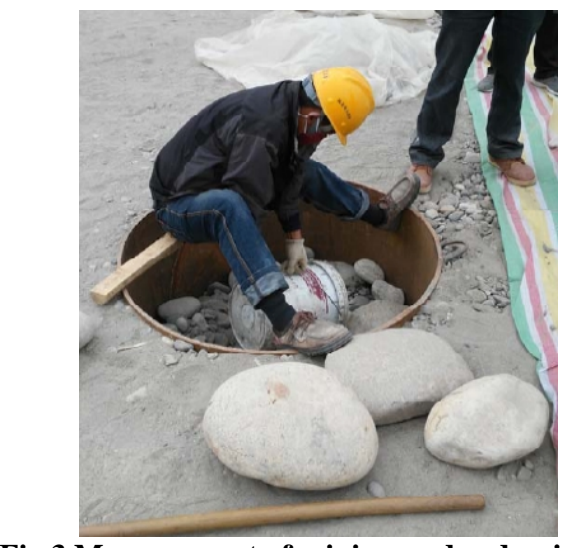

Fig.3 Measurement of minimum dry density by loose filling method

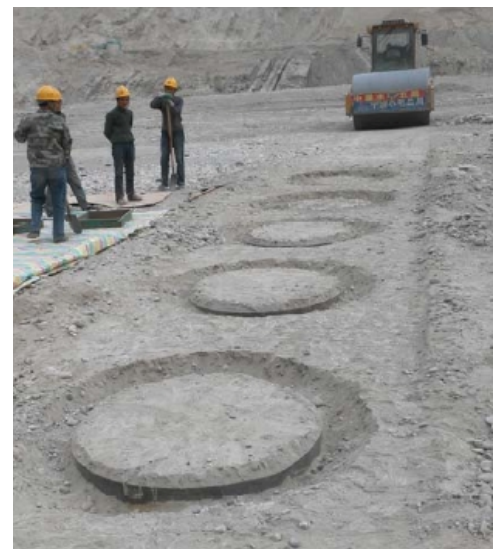

Fig.4 Test of maximum dry density after completion of strong vibratory rolling

\section{Test results and analysis}

The relative density of gravel soil is defined by the following formula ${ }^{[3]}$ :

$$
D_{r}=\frac{\rho_{d \max }\left(\rho_{d}-\rho_{d \min }\right)}{\rho_{d}\left(\rho_{d \max }-\rho_{d \min }\right)}
$$

In the formula: $D_{r}$ is the relative density, $\rho_{d \text { max }}$ is the maximum dry density, $\rho_{d \text { min }}$ is the minimum dry density, $\rho_{d}$ is the actual dry density of the soil, the latter three units are the same.

At first, the maximum and minimum dry density of five kinds of soil materials (design average gradation curve; upper envelope gradation curve; lower envelope gradation curve; upper average 
gradation curve and lower average gradation curve) with different gradation (coarse grain content) were tested, according to the test results, it can be determined that the optimum coarse grain content is basically the same as that of the design lower average gradation curve, so the soil material gradation of the maximum value test is consistent with the design lower average gradation curve. The test results of maximum and minimum dry density of soil with different gradation (coarse grain content) are shown in table1. As can be seen from table1, the maximum dry density of the test gravel soil is between $2.293 \mathrm{~g} / \mathrm{cm}^{3} \sim 2.442 \mathrm{~g} / \mathrm{cm}^{3}$ within the design envelope range, when the coarse grain content is $76.9 \%$, the dry density of the soil (lower average gradation curve) reached the maximum value; the minimum dry density is between $1.905 \mathrm{~g} / \mathrm{cm}^{3} \sim 2.068 \mathrm{~g} / \mathrm{cm}^{3}$, and the maximum value is also obtained when the coarse grain content is $76.9 \%$ (lower average gradation curve). The test result of the maximum and minimum dry density of the optimum coarse grain content soil is basically the same with that of the initial test, the two can verify each other, which indicates that the test process is well controlled and the test results are credible.

Tab.1 Test results of maximum and minimum dry density of soil with different gradation (coarse grain content)

\begin{tabular}{ccccccccc}
\hline & $\mathrm{P}_{5}(\%)$ & & 58.0 & 64.9 & 70.9 & 76.9 & 76.9 (check) & 83.7 \\
\hline \multirow{2}{*}{$\rho_{\text {dimax }}$} & & first & 2.305 & 2.365 & 2.416 & 2.443 & 2.441 & 2.378 \\
$\left(\mathrm{~g} / \mathrm{cm}^{3}\right)$ & second & 2.280 & 2.363 & 2.419 & 2.440 & 2.443 & 2.370 \\
& average & 2.293 & 2.364 & 2.418 & 2.441 & 2.442 & 2.374 \\
\hline \multirow{2}{*}{$\rho_{\text {dimin }}$} & first & 1.890 & 2.015 & 2.053 & 2.066 & 2.069 & 2.002 \\
$\left(\mathrm{~g} / \mathrm{cm}^{3}\right)$ & second & 1.920 & 1.985 & 2.057 & 2.067 & 2.066 & 1.993 \\
& average & 1.905 & 2.000 & 2.055 & 2.067 & 2.068 & 1.998 \\
\hline
\end{tabular}

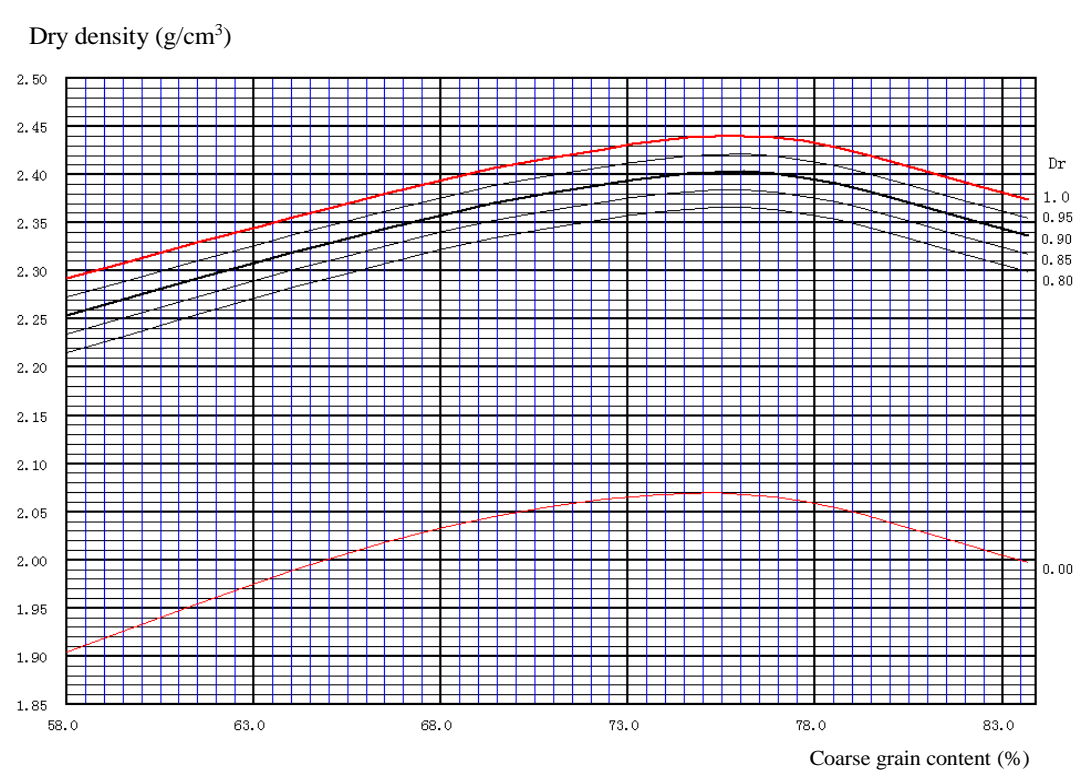

Fig.5 Three factors chart of relative density $\rho_{\mathrm{d}}-\mathrm{P}_{5}-\mathrm{D}_{\gamma}$ of gravel soil of Yulongkashi

Based on the test results, the three factors chart of gravel soil $\rho_{d}-P_{5}-D_{\gamma}$ (dry density, coarse grain content, relative density) in Yulongkashi was drawn, as shown in figure5. In the three factors chart, the maximum as well as minimum dry density and the absolute dry density value corresponding to different relative densities of soil with different gradation (coarse grain content) are reflected, which can be used as the evaluation standard for evaluating the rolling quality of gravel soil.

As can be seen from table1 and figure5:

(1) Compared with the results of laboratory soil test with similar engineering characteristics from the total value ${ }^{[3]}$, the maximum dry density test result of this field test is much higher than that of laboratory test, the laboratory test is affected by the size of the test equipment (density bucket) and the energy of the compaction equipment, the test results are much lower than the actual test results, without reasonable epitaxial treatment, it is difficult to evaluate the compaction quality of subsequent prototype soil, the field test overcomes the shortcoming that the scaling effect of 
laboratory test materials and compaction energy can not reflect the actual situation of the field, and the test results are more reliable.

(2) The maximum and minimum dry density of gravel soil increased first and then decreased with the increase of coarse grain content, there is an optimum coarse grain content, and at this point the gravel soil is most easily compacted, under the same compaction, the dry density of the soil is the largest.

(3) The optimum coarse grain content of gravel soil compaction quality in Yulongkashi corresponds to the design average lower envelope gradation curve, and which has a certain gap with design average gradation curve, which indicates that the proportion of the soil material in the optimum gradation is not high, attention should be paid to the dam design.

\section{Conclusions}

In view of the gravel soil of Yulongkashi high earth-rock fill dam, the field large-scale relative density test of original gradation was carried out, and the relative density characteristics of gravel soils were studied, the corresponding characteristic indexes used to guide the actual engineering design and construction quality control have been determined. The main conclusions are as follows:

(1) The field test overcomes the shortcoming that the scaling effect of laboratory test materials and compaction energy can not reflect the actual situation of the field, and the test results are more reliable, it can be directly applied to the quality evaluation of subsequent compaction construction without modification.

(2) Overall, the maximum dry density of field tests is much higher than that of similar laboratory soil tests.

(3) The maximum as well as minimum dry density of gravel soil increased first and then decreased with the increase of coarse grain content

(4) The maximum as well as minimum dry density and the absolute dry density value corresponding to different relative densities of soil with different gradation (coarse grain content) are reflected by the three factors chart, which can be used as the evaluation standard for evaluating the rolling quality of gravel soil.

\section{Acknowledgements}

This work was financially supported by the National Key Research and Development Program of China (2017YFC0404902), the National Natural Science Foundation of China (Grant No. 51509272), the special scientific research foundation of China Institute of Water Resources and Hydropower Research (GE0145B292017), the Public Service Sector R\&D Project of Ministry of Water Resource of China (Grant No. 201501035).

\section{References}

[1] Pan Z., Zhu J., Fang Z. Study on Relative Density Effects on Strength Behaviour of Sand-Gravel Material by Triaxial Test [J]. Yellow River, 2016, 38(2): 130-133.

[2] Tian S. Determining the Max. Dry Density of Coarse Grained Sandy Gravels by the Method of Curve-Fitting with Asymptotic Line [J]. Chinese Journal of Geotechnical Engineering, 1992, 14(1): 35-43.

[3] Gou Q. Research and application of the engineering properties of coarse-grained soil [M]. Zhengzhou: Yellow River Water Conservancy Press, 1984.

[4] Sun M., Zhu J., Shen K., et al. Density effects on strength and deformation behaviour of sandy gravel [J]. Hydro-Science and Engineering, 2015, (4): 43-47. 